\title{
(Un)distorted diffeomorphisms in different regularities
}

\author{
Andrés Navas*
}

\begin{abstract}
We build the first examples of diffeomorphisms that are distorted in a group of $C^{1}$ diffeomorphisms yet undistorted in the corresponding group of $C^{2}$ diffeomorphisms. This explicit construction is performed for the closed interval.
\end{abstract}

Recall that an element $f$ in a group $G$ is said to be distorted if there exists a finitely generated subgroup $\Gamma \subset G$ that contains $f$ such that

$$
\lim _{n \rightarrow \infty} \frac{\left\|f^{n}\right\|_{\Gamma}}{n}=0
$$

Here, $\|\cdot\|_{\Gamma}$ stands for the word-length of group elements with respect to any fixed finite generating system of $\Gamma$ (the definition of distortion elements above is independent of this choice, since any two such word-lengths are bi-Lipschitz equivalent). It is worth pointing out that the limit involved in this definition always exists, due to the (easy to check) subadditive inequality

$$
\left\|f^{m+n}\right\| \leq\left\|f^{n}\right\|+\left\|f^{m}\right\|
$$

and the well-known result commonly called Fekete's Lemma [12].

This notion was introduced by Gromov in [16]. Over the last years, it has attracted the attention of people not only from group theory and geometry, but also dynamics (see for instance [14, 18]). In this direction, it has been successfully used to exhibit obstructions to group actions on manifolds. Nevertheless, in most of the literature, the fact that groups of diffeomorphisms behave differently in distinct regularities is not considered. In this note we would like to address the following natural question:

Question. Given a compact manifold $M$ and integers $1 \leq r<s$, does there exist a $C^{s}$ diffeomorphism that is distorted in $\operatorname{Diff}_{+}^{r}(M)$ yet undistorted in $\operatorname{Diff}_{+}^{s}(M)$ ?

It is very likely that the answer to this question is affirmative. However, it seems hard to prove it using standard dynamical methods, such as genericity type arguments in a wellchosen subset of diffeomorphisms. Namely, as it was pointed out in [6], diffeomorphisms of positive topological entropy are undistorted in the group of $C^{1}$ diffeomorphisms (see the Appendix 2 for a discussion of this). Therefore, the question above involves zero-entropy diffeomorphisms, which are difficult to tackle. (Relevant recent progress on this topic that may be useful in this setting includes [7, 13.)

${ }^{*}$ Funded by Fondecyt Project 1200114 
Prototypes of zero-entropy maps are homeomorphisms of 1-manifolds, as it is widely known (see again Appendix 2 on this). In this work, we restrict to this framework and we provide a first result in smooth categories concerning the Question above.

Main Theorem. There exist $C^{\infty}$ diffeomorphisms of the closed interval that are $C^{1}$ distorted yet $C^{2}$-undistorted.

It is worth stressing that an analogous result holds in lower regularity. Indeed, every homeomorphism of the sphere (in any dimension) is $C^{0}$-distorted, yet it cannot be $C^{1}$ distorted if it is a $C^{1}$ diffeomorphism that admits hyperbolic periodic points (see [6]). It is easy to prove a similar statement for homeomorphisms of the interval (just use the fact that every nontrivial interval homeomorphism is conjugate to its square [15]).

To prove our result we put together two different ideas. On the one hand, in $\S 1$, we use the recently introduced notion of asymptotic distortion to ensure that diffeomorphisms in a suitable family are $C^{2}$ undistorted. On the other hand, in $\S 2$, we use methods arising in the study of centralizers to build finitely many $C^{1}$ diffeomorphisms that generate groups in which the above examples arise as distorted elements.

The construction that we develop gives us almost sharp examples in relation to the degree of distortion. On the one hand, our $C^{\infty}$ diffeomorphisms $f$ are almost logarithmically distorted, in the sense that $\left\|f^{n}\right\|$ growths slightly faster than logarithmically in $n$. On the other hand, no nontrivial $C^{1}$ diffeomorphism can be more than logarithmically distorted. See $\S 3$ for the details on this.

It would be certainly worthwhile to improve our methods so that the resulting diffeomorphisms are also distorted in the group of $C^{1+\alpha}$ diffeomorphisms for every $\alpha<1$. (Compare [2, 8.) This would obviously require more accurate estimates that we plan to develop in a complementary (more technical) work.

\section{On the asymptotic distortion of $C^{2}$ distorted diffeo- morphisms}

All maps in this note will be assumed to be orientation preserving.

Given a $C^{1+b v}$ diffeomorphism $f$ of a compact 1-manifold (either the circle or the closed interval), we denote by $\operatorname{var}(\log D f)$ the total variation of the logarithm of its derivative. (In this notation, " $b v$ " stands for "bounded variation".) The asymptotic distortion of $f$, denoted $\operatorname{dist}_{\infty}(f)$, is defined as the limit

$$
\lim _{n \rightarrow \infty} \frac{\operatorname{var}\left(\log D f^{n}\right)}{n} .
$$

This notion was introduced in [22] with a focus on circle diffeomorphisms, and later studied in [10] for diffeomorphisms of the interval. The relation with distorted elements is clear: if $f$ is distorted in the group of $C^{1+b v}$ diffeomorphisms, then its asymptotic distortion must vanish (this directly follows from the subaditivity property of $\operatorname{var}(\log D(\cdot))$; see [10, Corollary 4]). 
A nice family of diffeomorphisms with positive asymptotic distortion may be described as follows: Start with a $C^{1+b v}$ diffeomorphism of $[0,1]$ with vanishing asymptotic distortion and no fixed point in ]0,1[. Let $I$ be a fundamental domain for the action of $f$, that is, an open interval with endpoints $x_{0}, f\left(x_{0}\right)$ for a certain $\left.x_{0} \in\right] 0,1\left[\right.$. Let $g$ be any nontrivial $C^{1+b v}$ diffeomorphism of ]0,1[ supported on $I$. Then the diffeomorphism $\bar{f}:=f g$ is undistorted in $\operatorname{Diff}_{+}^{1+b v}([0,1])$, hence in $\operatorname{Diff}_{+}^{2}([0,1])$; see [10, Lemmas 2.2 and 7.2$]$.

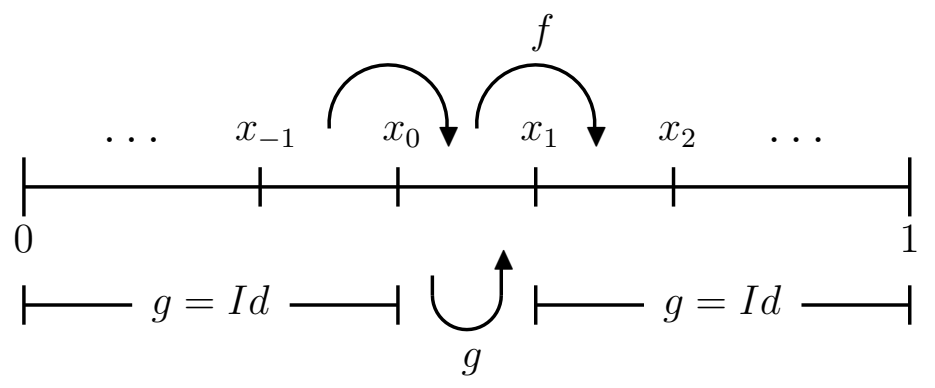

If $f$ is $C^{\infty}$ tangent to the identity at the endpoints, we may extend it to a larger interval (say, $[-1,2]$ ) by the identity outside $[0,1]$. It follows from the definition that this new diffeomorphism still has zero asymptotic distortion. Similarly, the extension of $\bar{f}$ has nonzero asymptotic distortion, and it is hence undistorted in $\operatorname{Diff}_{+}^{2}([-1,2])$.

Remark. Starting with the seminal work of Rosendal [28], over the last years there has been a lot of interest in understanding the geometry of (big) topological groups. In this setting, the word-metric is considered with respect to neighborhoods of the identity. The notion of distorted element that arises this way is weaker that the one considered in this paper, hence that of undistorted element is stronger. However, the proof above shows that $\bar{f}$ is still undistorted in this more restricted sense.

\section{How to force $C^{1}$ distortion}

We next explain how to perform the construction above so that the resulting diffeomorphism $\bar{f}$ of $[-1,2]$ is $C^{1}$ distorted. This will be accomplished in several steps.

Building the starting diffeomorphism $f$. Start with the vector fields $\hat{\mathcal{X}}$ and $\mathcal{X}$ on the real line whose time-1 maps are, respectively,

$$
\hat{F}:=\hat{\mathcal{X}}^{1}: x \mapsto 2 x \quad \text { and } \quad F:=\mathcal{X}^{1}: x \mapsto x+1 .
$$

It is well known that there exists a $C^{\infty}$ diffeomorphism $\left.\varphi: \mathbb{R} \rightarrow\right] 0,1\left[\right.$ such that $\hat{\mathcal{Y}}:=\varphi_{*}(\hat{\mathcal{X}})$ and $\mathcal{Y}:=\varphi_{*}(\mathcal{X})$ extend to the endpoints of $[0,1]$ as infinitely flat vector fields. (See for instance [24, 29].) Let us denote $\hat{f}:=\hat{\mathcal{Y}}^{1}$ and $f:=\mathcal{Y}^{1}$, which we view as diffeomorphisms of $[-1,2]$ that coincide with the identity outside $[0,1]$. It follows from [10] that $f$ has vanishing 
asymptotic distortion. Actually, $f$ is distorted in the group $\langle\hat{f}, f\rangle$. Indeed, the (BaumslagSolitar) relation $\hat{f} f \hat{f}^{-1}=f^{2}$ implies that, for each $k \geq 1$,

$$
\hat{f}^{i} f \hat{f}^{-i}=f^{2^{i}}
$$

As a consequence, $\left\|f^{n}\right\|$ is of order at most $O(\log (n))$. (Actually, it is of order $O(\log (n))$; this is very well known because of the Baumslag-Solitar group structure, but also follows from $\S 3$ below.)

Let $x_{0}:=\varphi(0)$ and, for each $n \in \mathbb{Z}$, let $x_{n}:=f^{n}\left(x_{0}\right)$. Notice that $x_{n}=\varphi(n)$. Denote also $x_{-1 / 2}:=\varphi(-1 / 2)$ and $x_{-3 / 4}:=\varphi(-3 / 4)$.

Building the diffeomorphism $g$. Let us denote by $\varphi_{0}$ the affine diffeomorphism sending $\left[x_{0}, x_{1}\right]$ onto $[0,1]$. Let $g:=\varphi_{0}^{-1} f \varphi_{0}$. This can be extended to $[-1,2]$ by the identity outside $\left[x_{0}, x_{1}\right]$. As above, we let $\bar{f}:=f g$.

A first estimate to prove distortion. Notice that

$$
\bar{f}^{n} f=(f g)^{n} f=f^{n+1}\left(f^{-n} g f^{n}\right)\left(f^{-(n-1)} g f^{n-1}\right) \cdots\left(f^{-2} g f^{2}\right)\left(f^{-1} g f\right),
$$

hence

$$
f^{-n}\left(f^{-1} \bar{f}^{n} f\right)=\left(f^{-n} g f^{n}\right)\left(f^{-(n-1)} g f^{n-1}\right) \cdots\left(f^{-2} g f^{2}\right)\left(f^{-1} g f\right) .
$$

Let us denote by $h_{n}$ this diffeomorphism. Clearly, it is supported on $\left[x_{-n}, x_{0}\right]$. Moreover, since $f$ is distorted in $\langle\hat{f}, f\rangle$, if we show that $\left\|h_{n}\right\|$ is $o(n)$ along an increasing (infinite) sequence of integers, then the same will be true for $\left\|f^{-1} \bar{f}^{n} f\right\|$, hence for $\left\|\bar{f}^{n}\right\|$. This will imply that $\bar{f}$ is a distorted element.

Building two auxiliary diffeomorphisms. We define two diffeomorphisms $\hat{h}$ and $h$ as follows:

(i) They act by the identity outside $[0,1]$.

(ii) On each interval $f^{n}\left(\left[x_{0}, x_{1}\right]\right)$, the diffeomorphism $\hat{h}$ (resp. $h$ ) coincides with the $s_{n}$-time map (resp. $t_{n}$-time map) of the flow of the vector field $f_{*}^{n}\left(\varphi_{0}^{*}(\hat{\mathcal{Y}})\right)\left(\operatorname{resp} . f_{*}^{n}\left(\varphi_{0}^{*}(\mathcal{Y})\right)\right)$.

Here, $s_{n}$ and $t_{n}$ are sequences of real numbers to be defined. According to [9, Lemma 4.1]1, in order to ensure that $\hat{h}$ and $h$ are $C^{1}$ at the endpoints (and hence $C^{1}$ diffeomorphisms), it is necessary and sufficient to impose the conditions $s_{n} \rightarrow 0$ and $t_{n} \rightarrow 0$ as $n \rightarrow \pm \infty$. In practice, we will choose these sequences as follows:

(iii) If $2^{i-1} \leq n<2^{i}$ for a certain positive even integer $i$, we let

$$
s_{n}:=\log _{2}\left(1-\frac{1}{\sqrt{\ell_{i / 2}}}\right) \quad \text { and } \quad t_{n}:=\frac{1}{\sqrt{\ell_{i / 2}}}
$$

\footnotetext{
${ }^{1}$ Related results appear in [3, 11]. For the reader's convenience, in the Appendix 1, we give a direct and simple proof that does not rely on Kopell's (difficult) estimates.
} 
where $\ell_{j}$ is any prescribed sequence of positive integers diverging to infinity.

(iv) For any other value of $n$, we let $s_{n}=t_{n}:=0$.

An extra diffeomorphism to control supports. Finally, we let $\psi$ be a $C^{\infty}$ diffeomorphism of $[-1,2]$ such that:

(v) $\psi$ coincides with the identity on $\left[x_{-1 / 2}, x_{0}\right]$.

(vi) $\psi\left(x_{-3 / 4}\right)=0$ and $\psi\left(x_{1}\right)=1$.

Computations below are made in the group $\Gamma:=\langle\hat{f}, f, g, \hat{h}, h, \psi\rangle\left(\right.$ contained in Diff $\left.{ }_{+}^{1}([-1,2])\right)$.

On the conjugates of $h$ and $\hat{h}$. Fix an integer $n$ of the form $2^{i}$, where $i$ is a large even number. We start by considering the conjugate maps $f^{-n} h f^{n}$, later $\hat{f}^{-i} f^{-n} h f^{n} \hat{f}^{i}$, and finally $\psi \hat{f}^{-i} f^{-n} h f^{n} \hat{f}^{i} \psi^{-1}$. Since

$$
\operatorname{supp}(h) \subset\left[0, x_{2^{i-2}}\right] \cup\left[x_{2^{i-1}}, x_{2^{i}}\right] \cup\left[x_{2^{i+1}}, 1\right],
$$

we have

$$
\operatorname{supp}\left(f^{-n} h f^{n}\right) \subset\left[0, x_{2^{i-2}-2^{i}}\right] \cup\left[x_{2^{i-1}-2^{i}}, x_{2^{i}-2^{i}}\right] \cup\left[x_{2^{i+1}-2^{i}}, 1\right]
$$

that is,

$$
\operatorname{supp}\left(f^{-n} h f^{n}\right) \subset\left[0, x_{2^{i-2}-2^{i}}\right] \cup\left[x_{-2^{i-1}}, x_{0}\right] \cup\left[x_{2^{i}}, 1\right] .
$$

Since $\left(2^{i-2}-2^{i}\right) / 2^{i}=-3 / 4$ and $-2^{i-1} / 2^{i}=-1 / 2$, this implies

$$
\operatorname{supp}\left(\hat{f}^{-i} f^{-n} h f^{n} \hat{f}^{i}\right) \subset\left[0, x_{-3 / 4}\right] \cup\left[x_{-1 / 2}, x_{0}\right] \cup\left[x_{1}, 1\right] .
$$

Finally, the properties (v) and (vi) imposed to $\psi$ imply

$$
\operatorname{supp}\left(\psi \hat{f}^{-i} f^{-n} h f^{n} \hat{f}^{i} \psi^{-1}\right) \subset[-1,0] \cup\left[x_{-1 / 2}, x_{0}\right] \cup[1,2] .
$$

Similarly,

$$
\operatorname{supp}\left(\hat{f}^{-i} f^{-n} \hat{h} f^{n} \hat{f}^{i}\right) \subset\left[0, x_{-3 / 4}\right] \cup\left[x_{-1 / 2}, x_{0}\right] \cup\left[x_{1}, 1\right]
$$

and

$$
\operatorname{supp}\left(\psi \hat{f}^{-i} f^{-n} \hat{h} f^{n} \hat{f}^{i} \psi^{-1}\right) \subset[-1,0] \cup\left[x_{-1 / 2}, x_{0}\right] \cup[1,2]
$$

$\boldsymbol{A}$ commutator of conjugates of $h$ and $\hat{h}$. Let us denote $a_{n}:=\hat{f}^{-i} f^{-n} h f^{n} \hat{f}^{i}$ and $b_{n}:=\psi \hat{f}^{-i} f^{-n} \hat{h} f^{n} \hat{f}^{i} \psi^{-1}$. We next compute $c_{n}:=\left[a_{n}, b_{n}\right]=a_{n}\left(b_{n} a_{n} b_{n}^{-1}\right)^{-1}$. We notice that:

- The intervals $[-1,0]$ and $[1,2]$ are invariant under both $a_{n}$ and $b_{n}$; besides, $a_{n}$ equals the identity therein. Therefore, on $[-1,0] \cup[1,2]$, the map $c_{n}$ acts trivially.

- The intervals $\left[0, x_{-1 / 2}\right]$ and $\left[x_{0}, 1\right]$ are invariant under both $a_{n}$ and $b_{n}$; besides, $b_{n}$ equals the identity therein. Therefore, on $\left[0, x_{-1 / 2}\right] \cup\left[x_{0}, 1\right]$, the map $c_{n}$ acts trivially as well. 
- By property (v) above, the restriction of $b_{n}$ to $\left[x_{-1 / 2}, x_{0}\right]$ coincides with that of $\hat{f}^{-i} f^{-n} \hat{h} f^{n} \hat{f}^{i}$. Therefore, the restriction of $c_{n}$ to this interval equals that of

$$
\hat{f}^{-i} f^{-n}\left(h \hat{h} h^{-1} \hat{h}^{-1}\right) f^{n} \hat{f}^{i} .
$$

Retrieving $h_{n}$ as a power of a commutator. Recall that

$$
h_{n}:=f^{-n}\left(f^{-1} \bar{f}^{n} f\right)=\left(f^{-n} g f^{n}\right)\left(f^{-(n-1)} g f^{n-1}\right) \cdots\left(f^{-2} g f^{2}\right)\left(f^{-1} g f\right) .
$$

We claim that, for $n=2^{i}$,

$$
h_{n / 2}=\left(\hat{f}^{i} c_{n} \hat{f}^{-i}\right)^{\ell_{i / 2}} .
$$

Indeed, by the discussion above, $\hat{f}^{i} c_{n} \hat{f}^{-i}$ is supported on $\hat{f}^{i}\left(\left[x_{-1 / 2}, x_{0}\right]\right)=\left[x_{-2^{i-1}}, x_{0}\right]$, which coincides with the support of $h_{n / 2}=h_{2^{i-1}}$. Moreover,

$$
\hat{f}^{i} c_{n} \hat{f}^{-i}=\left(f^{-n} h f^{n}\right)\left[\left(f^{-n} \hat{h} f^{n}\right)\left(f^{-n} h^{-1} f^{n}\right)\left(f^{-n} \hat{h}^{-1} f^{n}\right)\right]
$$

Furthermore, by (ii) and (iii), on each interval $\left[x_{k}, x_{k+1}\right]$, with $-2^{i-1} \leq k<0$, the map $\hat{h}$ (resp. $h)$ arises as the time- $s$ (resp. time- $t$ ) map of the flow of $f_{*}^{k}\left(\varphi_{0}^{*}(\hat{\mathcal{Y}})\right)\left(\operatorname{resp} . f_{*}^{k}\left(\varphi_{0}^{*}(\mathcal{Y})\right)\right)$, where

$$
s=\log _{2}\left(1-\frac{1}{\sqrt{\ell_{i / 2}}}\right) \quad \text { and } \quad t=\frac{1}{\sqrt{\ell_{i / 2}}} .
$$

The Lie-algebra relation between $\hat{\mathcal{Y}}$ and $\mathcal{Y}$ then implies that, on each such interval $\left[x_{k}, x_{k+1}\right]$, the commutator $\hat{f}^{i} c_{n} \hat{f}^{-i}=\left(f^{-n} h f^{n}\right)\left[\left(f^{-n} \hat{h} f^{n}\right)\left(f^{-n} h^{-1} f^{n}\right)\left(f^{-n} \hat{h}^{-1} f^{n}\right)\right]$ coincides with the time- $r$ map of the flow of $f_{*}^{k}(\mathcal{Y})$, where

$$
r=\left(1-2^{s}\right) t=\frac{1}{\ell_{i / 2}}
$$

Since the restriction of $h_{n}$ to $\left[x_{k}, x_{k+1}\right]$ is nothing but the time-1 map of the flow of $f_{*}^{k}(\mathcal{Y})$, this implies our claim.

A final estimate to prove distortion. Since $h_{n / 2}=\left(\hat{f}^{i} c_{n} \hat{f}^{-i}\right)^{\ell_{i / 2}}$, we have

$$
\left\|h_{n / 2}\right\| \leq \ell_{i / 2}\left\|\hat{f}^{i} c_{n} \hat{f}^{-i}\right\| \leq \ell_{i / 2}\left[2 i+\left\|c_{n}\right\|\right] \leq 2 \ell_{i / 2}\left[i+\left\|a_{n}\right\|+\left\|b_{n}\right\|\right]
$$

Using the definitions of $a_{n}=\hat{f}^{-i} f^{-n} h f^{n} \hat{f}^{i}$ and $b_{n}=\psi \hat{f}^{-i} f^{-n} \hat{h}^{n} \hat{f}^{i} \psi^{-1}$, this yields

$$
\left\|h_{n / 2}\right\| \leq 2 \ell_{i / 2}\left[i+\left(2 i+1+2\left\|f^{n}\right\|\right)+\left(2 i+3+2\left\|f^{n}\right\|\right)\right]=2 \ell_{i / 2}\left[5 i+4+4\left\|f^{n}\right\|\right] .
$$

Since $i \sim \log (n)$ and $\left\|f^{n}\right\|=O(\log (n))$, we conclude that

$$
\left\|h_{n / 2}\right\|=O\left(\ell_{i / 2} \log (n)\right) .
$$


Therefore, if we choose the sequence $\ell_{j}$ so that $\ell_{i / 2} \log (n)=o(n)$, we have that $\left\|h_{n}\right\|=o(n)$. As previously discussed, this implies that $\bar{f}$ is a distorted element of $\Gamma$.

Remark. It seems hard to completely describe the group $\Gamma$ above in algebraic terms. In particular, we do not know whether it contains free subgroups in two generators. However, it is easy to find elements for which Lemma 3.1 of [19] may be applied, thus showing that $\Gamma$ contains (many) copies of Thompson's group $F$; in particular, it is not solvable. Whether or not $C^{2}$-undistorted diffeomorphisms of $[0,1]$ may be distorted inside a solvable group of $C^{1}$ diffeomorphisms seems to be an interesting question. (Compare [4].)

\section{No super-exponential distortion}

Being distorted means that the word-length of powers grows sublinearly, but one may ask whether this growth is even weaker. In the explicit examples above, the computations show that $\bar{f}$ can be built as being slightly weaker than exponentially distorted. More precisely, for any sequence $k_{n}$ of positive integers diverging to infinity, $\left\|\bar{f}^{n}\right\|$ was showed to be of order $o\left(k_{n} \log (n)\right)$ inside an appropriate finitely generated group (Actually, this was proved along a subsequence of integers $n$ of the form $2^{i}$, but it is easy to ensure such a global behavior from this.) This is almost sharp, as proven next.

Proposition. Given a nontrivial $C^{1}$ diffeomorphism $\bar{f}$ inside a finitely generated group of diffeomorphisms of a closed interval, there exists a constant $A>0$ such that, for infinitely many integers $n \geq 0$,

$$
\left\|\bar{f}^{n}\right\| \geq A \log (n)
$$

Proof. This is essentially a restatement of an elementary observation from [27, pages 192193] that claims the following: If $\bar{f}$ is a nontrivial diffeomorphism of a closed interval, then there exists an increasing sequence of integers $n_{k}$ and points $x_{k}$ such that

$$
D \bar{f}^{n_{k}}\left(x_{k}\right) \geq n_{k} .
$$

Now, let $\bar{f}$ lie in a group of $C^{1}$ diffeomorphisms of the interval generated by finitely many elements $\left\{f_{1}, \ldots, f_{\ell}\right\}$, and let $C$ be the maximum possible value of all the $D f_{i}^{ \pm 1}$. If, for a fixed $k \geq 1$, we denote $m=m_{k}:=\left\|\bar{f}^{n_{k}}\right\|$, then

$$
\bar{f}^{n_{k}}=f_{i_{1}} \cdots f_{i_{m}}
$$

for certain $f_{i_{j}} \in\left\langle f_{1}, \ldots, f_{\ell}\right\rangle$. This obviously implies that $D \bar{f}^{n_{k}}(x) \leq C^{m}$ for all points $x$. Choosing $x=x_{k}$ we conclude that $n_{k} \leq C^{m}$, which implies $m \geq \log \left(n_{k}\right) / \log (C)$, that is,

$$
\left\|\bar{f}^{n_{k}}\right\| \geq \frac{\log \left(n_{k}\right)}{\log (C)},
$$

as claimed. 


\section{A remark on (un)distorted circle diffeomorphisms}

By gluing the endpoints of the interval, the previous example can be realized as a $C^{\infty}$ circle diffeomorphism with a single global fixed point. More generally, for any rational number $p / q$ (mod. 1), one can adapt the construction to build examples of $C^{\infty}$ circle diffeomorphisms of rotation number $p / q$ that are $C^{1}$ distorted yet $C^{2}$ undistorted. The situation for irrational rotation number is, however, less clear.

On the one hand, every $C^{2}$ (actually, $C^{1+b v}$ ) circle diffeomorphism $f$ of irrational rotation number $\rho$ is $C^{1}$ distorted. This follows as a combination of a slight modification of a theorem of Avila (indeed, his method of proof yields $C^{1}$ distortion provided $C^{1}$ recurrence is ensured) and a theorem of Herman [17] according to which these diffeomorphisms are recurrent, in the sense that $f^{q_{n}}$ converges to the identity in the $C^{1}$ topology as $n$ goes to infinity, where $p_{n} / q_{n}$ is the sequence of (best) rational approximations of $\rho$ (see [25] for a much simpler proof of this result).

On the other hand, we ignore whether a $C^{\infty}$ (or even $C^{2}$ ) diffeomorphism $f$ as above can be undistorted. In one direction, the main idea used in the construction of $C^{2}$ undistorted interval diffeomorphisms fails here, since the asymptotic distortion of such an $f$ always vanishes [22]. In the other direction, Avila's method for showing distortion fails not only because of the absence of recurrence in the $C^{2}$ topology, but also because another important tool of his proof, namely Mather's simplicity type results, is unavailable in the $C^{2}$ setting. However, it is worth mentioning that one can build $C^{1+b v}$ circle diffeomorphisms of irrational rotation number for which the asymptotic distortion is nonzero; these are hence undistorted in $\operatorname{Diff}_{+}^{1+b v}\left(\mathrm{~S}^{1}\right)$ (see [22, Remark 2] for the details).

\section{Appendix 1: A generalized version of Kopell's lemma}

In $\S 2$, we strongly used the result below. For $f$ of class $C^{2}$, this is Lemma 4.1 in [9]. Stated for $f$ of class $C^{1+\text { Lip }}$, this is Exercise 4.1.5 in [24]. Let us stress that we do not know whether this still holds for $f$ merely of class $C^{1+b v}$, which is the most general setting for the classical Kopell's lemma.

Lemma. Let $f$ be a $C^{1+\text { Lip }}$ diffeomorphism of $[0,1)$ such that $f(x)<x$ for all $x \in(0,1)$. Let $x_{0}$ be a point in $(0,1)$, and denote $x_{n}:=f^{n}\left(x_{0}\right)$. For each sequence $\left(g_{n}\right)$ of $C^{1}$ diffeomorphisms of $\left[x_{1}, x_{0}\right]$ that are tangent to the identity at the endpoints, the following conditions are equivalent:

(i) The sequence $\left(g_{n}\right)$ converges to the identity in the $C^{1}$ topology.

(ii) The diffeomorphism $g:\left(0, x_{0}\right] \rightarrow\left(0, x_{0}\right]$ whose restriction to $\left[x_{n+1}, x_{n}\right]$ coincides with $f^{n} g_{n} f^{-n}$ extends to a $C^{1}$ diffeomorphism of $\left[0, x_{0}\right]$ by letting $g(0)=0$.

Proof. Assuming (i), let us prove (ii). We need to show that, as $n$ goes to infinity, the derivative $D g(y)$ uniformly converges to 1 for $y \in\left[x_{n+1}, x_{n}\right]$. Using the relation $g=f^{n} g_{n} f^{-n}$ 
on $\left[x_{n+1}, x_{n}\right]$, we compute

$$
D g(y)=\frac{D f^{n}\left(g_{n} f^{-n}(y)\right)}{D f^{n}\left(f^{-n}(y)\right)} \cdot D g_{n}\left(f^{-n}(y)\right)=\frac{D f^{n}\left(z_{n}\right)}{D f^{n}\left(y_{n}\right)} \cdot D g_{n}\left(y_{n}\right)
$$

where $y_{n}:=f^{-n}(y)$ and $z_{n}:=g_{n}\left(y_{n}\right)$ both belong to $\left[x_{1}, x_{0}\right]$. Since $g_{n}$ converges to $I d$ in the $C^{1}$ topology, the factor $D g_{n}\left(y_{n}\right)$ converges to 1 as $n$ goes to infinity. Concerning the quotient of derivatives of $D f^{n}$, we first notice that, given $\varepsilon>0$, for $n$ large enough we have

$$
\frac{\left|z_{n}-y_{n}\right|}{\left|x_{0}-x_{1}\right|} \leq \frac{\left|z_{n}-y_{n}\right|}{\left|y_{n}-x_{1}\right|}=\left|\frac{g_{n}\left(y_{n}\right)-g_{n}\left(x_{1}\right)}{y_{n}-x_{1}}-1\right|=\left|D g_{n}\left(\xi_{n}\right)-1\right| \leq \varepsilon,
$$

where, in the previous computation, $\xi_{n}$ is a certain point in $\left[x_{1}, x_{0}\right]$. Now, for all $i \geq 0$, we have

$$
\frac{\left|f^{i}\left(z_{n}\right)-f^{i}\left(y_{n}\right)\right|}{\left|x_{n}-x_{n+1}\right|}=\frac{D f^{i}(q) \cdot\left(z_{n}-y_{n}\right)}{D f^{i}(p) \cdot\left(x_{0}-x_{1}\right)}
$$

for certain points $q=q_{i, n}$ and $p=p_{i, n}$ in $\left[x_{1}, x_{0}\right]$. Moreover, letting $C$ be the Lipschitz constant of $\log (D f)$ on $\left[0, x_{0}\right]$, we have, for all $i \geq 0$,

$$
\begin{aligned}
\left|\log \left(\frac{D f^{i}(q)}{D f^{i}(p)}\right)\right| & =\left|\sum_{j=0}^{i-1}\left[\log D f\left(f^{j}(q)\right)-\log D f\left(f^{j}(p)\right)\right]\right| \\
& \leq \sum_{j=0}^{i-1}\left|\log D f\left(f^{j}(q)\right)-\log D f\left(f^{j}(p)\right)\right| \\
& \leq \sum_{j=0}^{i-1} C\left|f^{j}(q)-f^{j}(p)\right| \\
& \leq C .
\end{aligned}
$$

According to (2) and (3), for a large-enough $n$, this implies

$$
\frac{\left|f^{i}\left(z_{n}\right)-f^{i}\left(y_{n}\right)\right|}{\left|x_{n}-x_{n+1}\right|} \leq \varepsilon e^{C} .
$$

Therefore,

$$
\begin{aligned}
\left|\log \left(\frac{D f^{n}\left(z_{n}\right)}{D f^{n}\left(y_{n}\right)}\right)\right| & =\left|\sum_{i=0}^{n-1}\left[\log D f\left(f^{i}\left(z_{n}\right)\right)-\log D f\left(f^{i}\left(y_{n}\right)\right)\right]\right| \\
& \leq \sum_{i=0}^{n-1}\left|\log D f\left(f^{i}\left(z_{n}\right)\right)-\log D f\left(f^{i}\left(y_{n}\right)\right)\right| \\
& \leq \sum_{i=0}^{n-1} C\left|f^{i}\left(z_{n}\right)-f^{i}\left(y_{n}\right)\right| \\
& \leq C \varepsilon e^{C} \sum_{i=0}^{n-1}\left|x_{n}-x_{n+1}\right| \\
& \leq C \varepsilon e^{C} .
\end{aligned}
$$


This implies that the value of the quotient in the expression (11) lies in $\left[e^{-\varepsilon C^{\prime}}, e^{\varepsilon C^{\prime}}\right]$ for $C^{\prime}:=C e^{C}$. Since $\varepsilon>0$ was arbitrary, this shows that, as $n$ goes to infinity, the value of $D g(y)$ uniformly converges to 1 for $y \in\left[x_{n+1}, x_{n}\right]$.

The proof that (ii) implies (i) roughly consists in reversing the arguments above. To begin with, just notice that $D g(0)$ must be equal to 1 , since $g$ fixes all the points $x_{n}$, which converge to the origin. The rest of the computations are straightforward and may be left to the reader.

\section{Appendix 2: A remark on entropy and distortion}

As it is cleverly noticed in [6], an application of Ruelle's inequality proves that $C^{1}$ diffeomorphisms of compact manifolds with positive topological entropy are undistorted. Here we extend this fact to bi-Lipschitz homeomorphisms.

Proposition. Every bi-Lipschitz homeomorphism of a compact manifold with positive topological entropy is undistorted (in the corresponding group of bi-Lipschitz homeomorphisms).

Proof. This relies on the Bowen-Kouchnirenko inequality [5, 21] for a $C^{1}$ diffeomorphism $f$ of a $d$-dimensional compact manifold:

$$
\left.h_{\mathrm{top}}(f) \leq d \cdot \max _{x} \log (\| D f(x)) \|\right) .
$$

Indeed, this inequality holds (with the very same proof) for bi-Lipschitz homeomorphisms. In this setting, the "max" should be replaced by an "essup", which is controlled by the Lipschitz constant of the map2:

$$
h_{\mathrm{top}}(f) \leq d \cdot \log (\operatorname{Lip}(f))
$$

Now, let $f$ be a bi-Lipschitz homeomorphism of a compact $d$-dimensional manifold that is distorted, and let $\Gamma$ be a finite generating system of a group of bi-Lipschitz homeomorphisms containing $f$ such that

$$
\lim _{n \rightarrow \infty} \frac{\left\|f^{n}\right\|_{\Gamma}}{n}=0
$$

If $\ell_{n}:=\left\|f^{n}\right\|_{\Gamma}$, then there exist $f_{i_{1}}, \ldots, f_{i_{\ell_{n}}}$ in $\Gamma$ such that $f^{n}=f_{i_{1}} \cdots f_{i_{\ell_{n}}}$. If we denote by $L$ the maximum of the Lipschitz constants of the maps in $\Gamma$, this implies $\operatorname{Lip}\left(f^{n}\right) \leq L^{\ell_{n}}$. Thus,

$$
\log \left(\operatorname{Lip}\left(f^{n}\right)\right) \leq \ell_{n} \log (L)
$$

\footnotetext{
${ }^{2}$ This more general version allows reproving the well-known fact that homeomorphisms of (compact) 1-manifolds have zero entropy. Indeed, an elementary argument given in [23] establishes that these homeomorphisms are topologically conjugate to bi-Lipschitz homeomorphisms with Lipschitz constant as close to 1 as desired.
} 
By the Bowen-Kournichenko inequality, this yields

$$
h_{\mathrm{top}}(f)=\frac{h_{\mathrm{top}}\left(f^{n}\right)}{n} \leq \frac{1}{n} d \cdot \ell_{n} \log (L),
$$

which implies that $h_{\text {top }}(f)=0$ by letting $n$ go to infinity.

Acknowledgments. I wish to thank Adrien Le Boudec, Sang-Hyun Kim and Christian Rosendal for their useful remarks, Jairo Bochi and Godofredo Iommi for a couple of references, and the referee for her/his many corrections.

\section{References}

[1] A. Avila. Distortion elements in $\operatorname{Diff}^{\infty}(\mathbb{R} / \mathbb{Z})$. Preprint (2008).

[2] H. BAik, S. Kim \& T. Koberda. Unsmoothable group actions on compact onemanifolds. Journal of European Mathematical Society 21 (2019), no. 8, 2333-2353.

[3] C. Bonatti \& É. Farinelli. Centralizers of $C^{1}$-contractions of the half line. Groups Geom. Dyn. 9 (2015), no. 3, 831-889.

[4] C. Bonatti, I. Monteverde, A. Navas \& C. Rivas. Rigidity for $C^{1}$ actions on the interval arising from hyperbolicity I: solvable groups. Math. Z. 286 (2017), 919-949.

[5] R. Bowen. Entropy for group endomorphisms and homogeneus spaces. Trans. of the AMS 135 (1971), 401-414.

[6] D. Calegari \& M. H. Freddman, with an appendix by Y. De Cornulier. Distortion in transformation groups. Geometry \& Topology 10 (2006), 267-293.

[7] S. Crovisier, E. Pujals \& C. Tresser. Mild dissipative diffeomorphisms of the disk with zero entropy. Preprint (2020).

[8] B. Deroin, V. Kleptsyn, \& A. Navas. Sur la dynamique unidimensionnelle en régularité intermédiaire. Acta Math. 199, Number 2 (2007), 199-262.

[9] S. Druck \& S. Firmo. Periodic leaves for diffeomorphisms preserving codimension one foliations. J. Math. Soc. Japan 55 (2003), no. 1, 13-37.

[10] H. Eynard-Bontemps \& A. NAvas. Mather invariant, conjugates, and distortion for diffeomorphisms of the interval. Preprint (2019).

[11] É. FARINELli. Conjugacy classes of diffeomorphisms of the interval in $C^{1}$-regularity. Fund. Math. 237 (2017), no. 3, 201-248.

[12] M. Fekete. Über die Verteilung der Wurzeln bei gewissen algebraischen Gleichungen mit ganzzahligen Koeffizienten. Mathematische Zeitschrift 17 (1923), 228-249.

[13] J. Franks \& M. HAndel. Entropy zero area preserving diffeomorphisms of $S^{2}$. Geometry $\&$ Topology 16 (2012), 2187-2284. 
[14] J. Franks \& M. Handel. Distortion elements in group actions on surfaces. Duke Math. J. 131 (2006), 441-468.

[15] É. GHys. Groups acting on the circle. L'Enseignement Mathématique 47 (2001), 329-407.

[16] M. Gromov. Asymptotic invariants of infinite groups, from: "Geometric group theory, Vol. 2 (Sussex, 1991)". London Math. Soc. Lecture Notes Ser. 182, Cambridge Univ. Press, Cambridge (1993) 1-295.

[17] M. Herman. Sur la conjugaison diffrentiable des difféomorphismes du cercle à des rotations. Publ. Math. de l'IHÉS 49 (1979), 5-233.

[18] S. Hurtado. Continuity of discrete homomorphisms of diffeomorphism groups. Geom. Topol. 19 (2015), no. 4, 2117-2154.

[19] S. H. Kim, T. Koberda \& Y. Lodha. Chain groups of homeomorphisms of the interval. Ann. Sci. Ec. Norm. Supérieure 4 (2019), 797-820.

[20] N. Kopell. Commuting diffeomorphisms. In Global Analysis (Proc. Sympos. Pure Math., Vol. XIV, Berkeley, Calif., 1968). AMS, Providence, R.I. (1970), 165-184.

[21] A. G. Kuchnirenko. An upper bound of the entropy of a classical dynamical system. Dokl. Akad. Nauk. SSSR 6 (1965), 37-38. Soviet. Math Doklady 6 (1965), 360-362.

[22] A. NAvAs. On conjugates and the asymptotic distortion of 1-dimensional $C^{1+b v}$ diffeomorphisms. Preprint (2018).

[23] A. Navas. Sur les rapprochements par conjugaison en dimension 1 et classe $C^{1}$. Compositio Math. 150 (2014), 1183-1195.

[24] A. Navas. Groups of Circle Diffeomorphisms. Chicago Lectures in Mathematics, Univ. of Chicago Press (2011).

[25] A. Navas \& M. Triestino. On the invariant distributions of $C^{2}$ circle diffeomorphisms of irrational rotation number. Math. Z. 274 (2013), no. 1-2, 315-321.

[26] D. Pixton. Nonsmoothable, unstable group actions. Trans. AMS 229 (1977), 259-268.

[27] L. Polterovich \& M. Sodin. A growth gap for diffeomorphisms of the interval. $J$. Anal. Math. 92 (2004), 191-209.

[28] C. Rosendal. Coarse Geometry of Topological Groups. Unpublished book.

[29] T. Tsuboi. $\Gamma_{1}$-structures avec une seule feuille. Astérisque 116 (1984), 222-234.

[30] T. Tsubor. Homological and dynamical study on certain groups of Lipschitz homeomorphisms of the circle. J. Math. Soc. Japan 47 (1995), 1-30.

Andrés Navas

Dpto. de Matemática y C.C., Univ. de Santiago de Chile (USACH)

Alameda 3363, Estación Central, Santiago, Chile

Email: andres.navas@usach.cl 